\title{
Dietary supplementation with lactose or artificial sweetener enhances swine gut Lactobacillus population abundance
}

\author{
Kristian Daly ${ }^{1}$, Alistair C. Darby ${ }^{1}$, Neil Hall ${ }^{1}$, Alexandra Nau ${ }^{2}$, David Bravo ${ }^{2}$ and \\ Soraya P. Shirazi-Beechey ${ }^{1 *}$ \\ ${ }^{1}$ Epithelial Function and Development Group, Department of Functional and Comparative Genomics, \\ Institute of Integrative Biology, University of Liverpool, Liverpool L69 7ZB, UK \\ ${ }^{2}$ Pancosma SA, Geneva, Switzerland
}

(Submitted 5 November 2012 - Final revision received 13 February 2013 - Accepted 21 February 2013)

\begin{abstract}
The commensal bacteria Lactobacillus are widely used as probiotic organisms conferring a heath benefit on the host. They have been implicated in promoting gut health via the stimulation of host immunity and anti-inflammatory responses, as well as protecting the intestinalmucosa against pathogen invasion. Lactobacilli grow by fermenting sugars and starches and produce lactic acid as their primary metabolic product. For efficient utilisation of varied carbohydrates, lactobacilli have evolved diverse sugar transport and metabolic systems, which are specifically induced by their own substrates. Many bacteria are also capable of sensing and responding to changes in their environment. These sensory responses are often independent of transport or metabolism and are mediated through membrane-spanning receptor proteins. We employed DNA-based pyrosequencing technology to investigate the changes in the intestinal microbiota of piglets weaned to a diet supplemented with either a natural sugar, lactose or an artificial sweetener (SUCRAM ${ }^{\circledR}$, consisting of saccharin and neohesperidin dihydrochalcone (NHDC); Pancosma SA). The addition of either lactose or saccharin/NHDC to the piglets' feed dramatically increased the caecal population abundance of Lactobacillus, with concomitant increases in intraluminal lactic acid concentrations. This is the first report of the prebiotic-like effects of saccharin/NHDC, an artificial sweetener, being able to influence the commensal gut microbiota. The identification of the underlying mechanism(s) will assist in designing nutritional strategies for enhancing gut immunity and maintaining gut health.
\end{abstract}

Key words: Intestine: Microbiota: Pig: Artificial Sweetener: Lactobacillus

The microbial colonisation of the gastrointestinal tract at birth, predominantly by lactic acid bacteria, including Lactobacillus, Bifidobacteria and Streptococcus, is of paramount importance to health and performance through the stimulation of gut function, immunity and maturation ${ }^{(1-4)}$. After weaning, the gut microbiota becomes more complex and is capable of fermenting indigestible dietary components to monocarboxylates, mainly acetate, propionate, butyrate and lactate. The absorption of acetate, propionate and butyrate by colonic epithelial cells provides a valuable energy source, with butyrate also regulating homeostasis of the colonic epithelium by controlling the expression of genes associated with the proliferation, differentiation and apoptosis of colonic epithelial cells ${ }^{(5-9)}$. Furthermore, the production of lactic acid reduces colonic $\mathrm{pH}$, thereby inhibiting pathogenic organisms ${ }^{(10)}$.
In today's commercial pig industry, piglets are weaned between 21 and $35 \mathrm{~d}$ of age ${ }^{(11)}$. Although early weaning increases the number of piglets born per annum, the sudden and major change in the diet greatly increases the risk of enteric disease, diarrhoea and malnutrition ${ }^{(11)}$. It has been suggested that the high susceptibility of early-weaned piglets to enteric disorders is due to disruption in the establishment of a stable intestinal microbiota, thereby allowing pathogenic bacteria to flourish and cause disease $\mathrm{e}^{(12-14)}$.

Nutritional strategies, designed to prevent enteric disorders and improve the health and growth of piglets, may have the potential to influence the gastrointestinal microbiota. These strategies commonly entail the inclusion of dietary supplements such as dairy products ${ }^{(15)}$, natural sugars ${ }^{(16,17)}$, artificial sweeteners $^{(18)}$, fermentable carbohydrates ${ }^{(19,20)}$ and even

Abbreviations: HC, basal hydrolysable carbohydrate diet; HC + L, basal hydrolysable carbohydrate diet supplemented with 5\% (w/w) lactose; HC $+\mathrm{S}$, basal hydrolysable carbohydrate diet supplemented with $0.015 \%$ (w/w) sucram (saccharin/NHDC); NHDC, neohesperidin dihydrochalcone; PTS, phosphoenolpyruvate:carbohydrate phosphotransferase system; SGLT1, $\mathrm{Na}^{+} /$glucose co-transporter 1.

*Corresponding author: S. P. Shirazi-Beechey, email spsb@liverpool.ac.uk 
probiotic micro-organisms ${ }^{(21)}$. However, the precise effects of these dietary supplements on the gastrointestinal microbiota have not been fully characterised. We employed 16S rDNAbased 454 pyrosequencing technology ${ }^{(22)}$ to assess the composition of caecal microbiota in piglets. The present brief study focuses on the effect of dietary supplementation of the piglets' feed with either lactose or an artificial sweetener (saccharin/NHDC) specifically on the population abundance of gut Lactobacillus.

Dietary lactose has previously been shown to act as a prebiotic, promoting the growth of beneficial commensal bacteria, such as Bifidobacteria and Lactobacillus, and improving gut health and growth performance in weaning piglets $^{(15,16)}$. Dietary supplementation with saccharin/NHDC $\left(\right.$ SUCRAM $^{\circledR}$ ), the only artificial sweetener that has approval for use as a feed additive in the European Union ${ }^{(23)}$, has also been shown to dramatically reduce enteric disease and to enhance growth performance in early-weaned piglets ${ }^{(18)}$. However, very few studies ${ }^{(24,25)}$ on the prebiotic-like effects of sweeteners on the gastrointestinal microbiota have been published.

In the present study, we observed significant increases in the caecal population abundance of Lactobacillus in response to the inclusion of artificial sweetener consisting of saccharine and NHDC in the piglets' feed. The mechanism(s) underlying the increased abundance of caecal Lactobacillus in response to dietary supplementation with saccharin/NHDC are presently unknown. Processes, such as the influence of host-derived factors upon the microbiota ${ }^{(26,27)}$ and/or the involvement of a Lactobacillus cell membrane-associated sensor for recognising artificial sweetener, are proposed to explain this observation.

\section{Methods}

\section{Animals and collection of samples}

Male and female suckling Landrace $\times$ Large White piglets aged $28 \mathrm{~d}$ were placed in pairs and housed in standard pens $\left(1.5 \mathrm{~m}^{2}\right.$, $12 \mathrm{~h}$ light-dark cycle and $26 \cdot 7^{\circ} \mathrm{C}$ ). A total of three groups, each consisting of eight animals, were weaned to and maintained on the following isoenergetic $(16 \cdot 76-16.82 \mathrm{~kJ} / \mathrm{g})$ diets for 2 weeks: group 1, a commercial wheat- and soya-based swine basal diet (Target Feeds Limited) containing $42 \%$ (w/w) hydrolysable carbohydrates (HC); group 2, the same basal diet but containing 5\% (w/w) lactose (in the form of dairy crest whey) $(\mathrm{HC}+\mathrm{L})$; group 3 , the same basal diet but supplemented with $0.015 \%(\mathrm{w} / \mathrm{w})$ SUCRAM $^{\circledR}$ (an artificial sweetener consisting of saccharin and neohesperidin dihydrochalcone $(\mathrm{HC}+\mathrm{S})$. All the animals had free access to food and water at all times and consumed the same amount of feed. They all remained healthy throughout the course of the feeding trial, and had no signs of enteric disturbances. After 2 weeks, the piglets were killed with an intravenous injection of pentobarbitone (200 mg Pentoject/ml; AnimalCare Limited) into the cranial vena cava (according to UK Home Office Schedule 1 regulations). National/institutional guidelines for the care and use of animals were followed, and all experiments were approved by the University of Liverpool Ethics Committee. Immediately post-mortem, caecal and rectal contents were removed, wrapped in aluminium foil and frozen in liquid $\mathrm{N}_{2}$. All samples were subsequently stored at $-80^{\circ} \mathrm{C}$ until used for microbial DNA extraction or capillary GC analysis.

\section{Extraction of bacterial DNA from caecal content samples}

Nucleic acid was extracted from the samples of caecal contents using the method outlined by Lin \& Stahl ${ }^{(28)}$ and described previously ${ }^{(29)}$. Approximately $1 \mathrm{~g}$ aliquots of frozen samples were transferred to screw-cap tubes containing SDS, Trisbuffered phenol (pH 8.0) (Sigma-Aldrich Company Limited) and sterile acid-washed glass beads. The samples were immediately homogenised using a mini beadbeater (Biospec Corporation, Stratech Scientific). The aqueous supernatant was then extracted with phenol-chloroform-isoamylalcohol and treated with DNase-free RNase A to remove contaminating RNA. Total DNA (primarily bacterial) was precipitated by the addition of sodium acetate and isopropanol. Purified DNA was resuspended in sterile Tris buffer and stored at $-80^{\circ} \mathrm{C}$. DNA integrity was assessed by agarose gel electrophoresis. We observed that rapid freezing of samples in liquid $\mathrm{N}_{2}$, followed by homogenisation in a buffer containing phenol, is an effective method for inactivating nuclease activity. This approach also avoids repeated freeze-thawing of samples that may be deleterious to the efficient isolation of DNA from Gram-negative microbes.

\section{PCR amplification of bacterial 16S rRNA genes (rDNA)}

Purified DNA was used as a template for PCR amplification of bacterial 16S rRNA genes using GS FLX + Titanium fusion primers, targeted to flanking regions of the $\mathrm{V} 1-\mathrm{V} 3$ loop of bacterial 16S rDNA, producing amplicons of approximately $500 \mathrm{bp}$ (sense 5'-CAGGCCTAACACATGCAAGTC-3'; antisense $5^{\prime}$-ATTACCGCGGCTGCTGG-3'). PCR cycling was kept to a maximum of eighteen cycles to avoid chimera production. Amplicons from each group of piglets, labelled by the inclusion of a Multiplex Identifier sequence tag, were pooled in equimolar amounts, and sequenced at the Centre for Genomics Research, University of Liverpool, on a 454 GS FLX + Titanium sequencing platform (Roche).

\section{Analysis of 454 GS FLX + sequence reads}

Post-sequencing, raw reads were de-multiplexed using the Multiplex Identifier sequence tags and corrected for PCR and sequencing artifacts using AmpliconNoise ${ }^{(30)}$ and ChimeraSlayer ${ }^{(31)}$. The reads were aligned using PyNAST and Greengenes ${ }^{(32,33)}$ and phylogenies calculated with FASTTREE $^{(34)}$. Operational taxonomic units were defined, using a similarity threshold of $97 \%$, with UCLUST $^{(35)}$ and taxonomy was assigned via RDP Classifier $2.2^{(36)}$ using Qiime version $1.5^{(37)}$ to implement analysis workflow. 


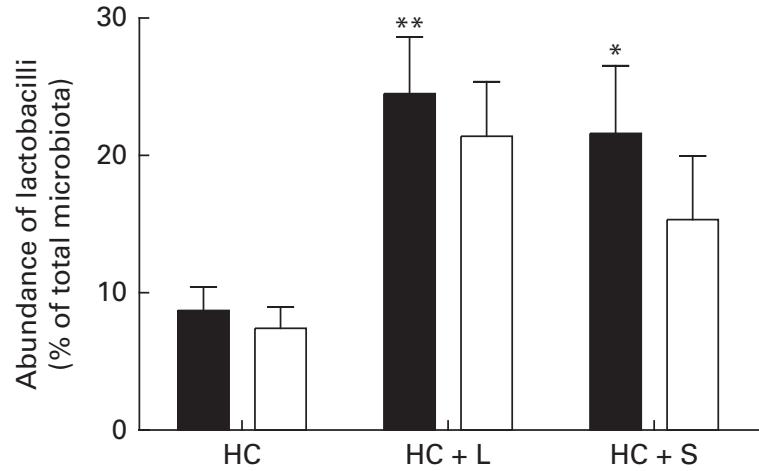

Fig. 1. Population abundance of caecal total lactobacilli $(\square)$ and Lactobacillus OTU4228 ( $\square$ ) (expressed as a percentage of the total microbiota) in piglets weaned to a basal hydrolysable carbohydrate $(\mathrm{HC})$ diet and a $\mathrm{HC}$ diet supplemented with $5 \%(\mathrm{w} / \mathrm{w})$ lactose $(\mathrm{HC}+\mathrm{L})$ or $0.015 \%(\mathrm{w} / \mathrm{w})$ saccharin/ NHDC $(\mathrm{HC}+\mathrm{S})$. Values are means, with their standard errors represented by vertical bars. Mean values were significantly different from those of the $\mathrm{HC}$ diet: ${ }^{\star} P<0.05,{ }^{*} P<0.01$.

\section{Measurement of caecal lactic acid concentrations}

The concentration of lactic acid in the caecal contents of piglets was measured as described previously ${ }^{(38)}$. Briefly, thawed caecal contents were centrifuged to remove particulates, and lactic acid was extracted from supernatants by the addition of concentrated $\mathrm{HCl}$ and diethyl ether. The diethyl ether layer extracts were then derivatised and lactic acid concentrations determined by capillary GC, quantified in relation to an internal standard.

\section{Statistical analysis}

Data are presented as means with their standard errors. Significance of differences was determined using one-way ANOVA with Bonferroni's multiple comparison test (GraphPad Prism 5; GraphPad Software, Inc.). Results were considered significant if $P<0.05$.

\section{Results}

\section{Effect of lactose on the population abundance of swine gut Lactobacillus}

Supplementation with the $\mathrm{HC}+\mathrm{L}$ diet resulted in a significant enhancement of the caecal Lactobacillus population (expressed as a percentage of the total number of sequences) from 8.7 (SEM 1.7) to 24.5 (SEM 4.1 ) \%, a $2 \cdot 8$-fold increase $(P<0.01$; Fig. 1). Furthermore, this increase was observed to be almost entirely due to one particular phylotype, designated Lactobacillus OTU4228, which increased from $7 \cdot 4$ (SEM 1.5) \% of the total microbiota in piglets weaned to the basal HC diet to $21 \cdot 4$ ( $\operatorname{sem} 4 \cdot 0) \%$ of the total microbiota in those weaned to the $\mathrm{HC}+\mathrm{L}$ diet $(P<0 \cdot 01 ;$ Fig. 1$)$.

Moreover, the measurements of lactic acid concentrations in the caecal contents of piglets weaned to the $\mathrm{HC}+\mathrm{L}$ diet showed lactic acid to be present at a concentration of $15 \cdot 2$ (SEM 1.8) $\mathrm{mm}$; a 10-fold increase compared with caecal lactic acid concentrations in piglets weaned to the basal HC diet (1.5 (SEM 0.2) mM) ( $P<0.001$; Fig. 2).

\section{Effect of saccharin/NHDC on the population abundance of swine gut Lactobacillus}

A significant enhancement of the relative population size of caecal Lactobacillus was similarly observed in piglets weaned to the $\mathrm{HC}+\mathrm{S}$ diet. In these piglets, Lactobacillus accounted for 21.6 (SEM 4.9) \% of the total microbiota (expressed as a percentage of the total number of sequences), compared with 8.7 (SEM 1.7 ) \% in piglets weaned to the basal HC diet. This represents an increase of $2 \cdot 5$-fold ( $P<0.05$; Fig. 1$)$. Again, this increase was observed to be almost solely due to an increase in the population of Lactobacillus OTU4228, which comprised $15 \cdot 3$ (SEM $4 \cdot 6) \%$ of the total microbiota in piglets weaned to the $\mathrm{HC}+\mathrm{S}$ diet $(P<0 \cdot 05$; Fig. 1$)$

Furthermore, caecal lactic acid concentrations in response to the inclusion of saccharin/NHDC were increased by $2 \cdot 1$-fold over concentrations of caecal lactic acid in piglets weaned to the basal HC $\operatorname{diet}(3.2$ (SEM 0.6$) \quad v .1 .5$ (SEM 0.2) mM, $P<0 \cdot 05$; Fig. 2).

\section{Discussion}

Lactobacilli are the predominant lactic acid bacteria found in the pig intestine and constitute a major proportion of the entire intestinal microbiota. As such, they are of particular importance to the maintenance of gut health. The presence and activity of lactobacilli have a stimulatory effect on both gut immunity and maturation, enhancing immune protection and reducing gastrointestinal inflammatory responses ${ }^{(39,40)}$. They also display antimicrobial activities that participate in host epithelial defence, such as reduction of colonic $\mathrm{pH}$ (through the production of lactic acid), protection against mucosal pathogen invasion and production of bacteriocins ${ }^{(9,41,42)}$.

We used PCR amplification of bacterial 16S rRNA gene sequences and subsequent 454 pyrosequencing to identify changes in swine gut microbiota in response to dietary supplementation. The average number of sequence reads per

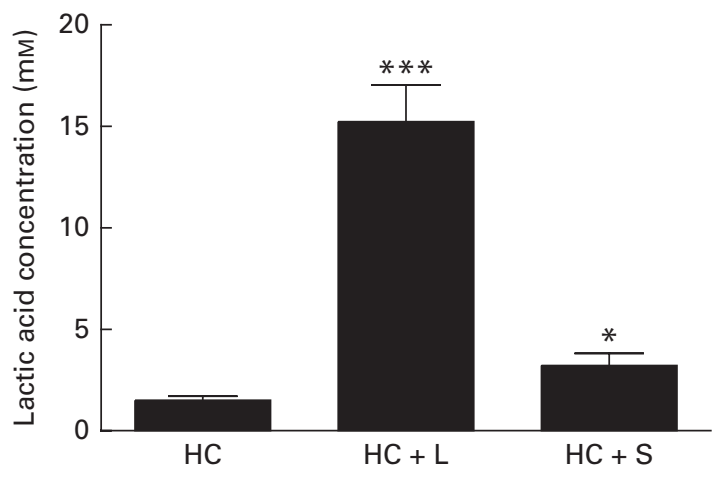

Fig. 2. Concentration of lactic acid in the caecal contents of piglets fed a basal hydrolysable carbohydrate diet $(\mathrm{HC})$ and a $\mathrm{HC}$ diet supplemented with $5 \%$ $(\mathrm{w} / \mathrm{w})$ lactose $(\mathrm{HC}+\mathrm{L})$ or $0.015 \%(\mathrm{w} / \mathrm{w})$ saccharin/NHDC $(\mathrm{HC}+\mathrm{S})$. Values are means, with their standard errors represented by vertical bars. Mean values were significantly different from those of the $\mathrm{HC}$ diet: ${ }^{*} P<0.05$, ${ }^{\star \star \star} P<0.001$. 
sample was in excess of 15000 and using a similarity threshold of $97 \%$ allowed classification of over 1000 phylotypes. Taxonomic assignment of these phylotypes showed that, in these piglets, the caecal microbiota is dominated by three major bacterial classes: Bacteroidia, Bacilli and Clostridia, which together contribute over $90 \%$ of all sequences. Major bacterial groups represented include Porphyromonas and Prevotella (Bacteroidia), Lactobacillus (Bacilli) and Ruminococcus, Lachnospira and Veillonella (Clostridia).

In the present study, which focuses exclusively on changes within the Lactobacillus populations, we report that there were significant enhancements in the relative population abundance of lactobacilli in the caecal contents of piglets in response to dietary supplementation with either a natural sugar, lactose or an artificial sweetener (saccharin/NHDC). The addition of lactose $(5 \%, \mathrm{w} / \mathrm{w})$ to the basal diet resulted in caecal Lactobacillus populations increasing from approximately $9 \%$ to over $24 \%$ of the total microbiota. Notably, supplementation of the basal diet with saccharin/NHDC (0.015\%, w/w; a 330-fold lower concentration than lactose) also increased caecal Lactobacillus populations to almost the same level (approximately $22 \%$ of the total microbiota) (Fig. 1). In parallel, caecal lactic acid concentrations $(1.5 \mathrm{~mm}$ in piglets weaned to the basal HC diet) increased 10-fold to over $15 \mathrm{~mm}$ in piglets weaned to the same diet containing lactose and 2 -fold to over $3 \mathrm{~mm}$ in piglets weaned to the diet containing saccharin/NHDC (Fig. 2).

Interestingly, the increase in Lactobacillus abundance observed in the caecal contents of piglets weaned to diets containing either lactose or artificial sweetener is not a general enhancement in all Lactobacillus populations present in the pig caecum. In fact, one particular phylotype, designated Lactobacillus OTU4228, is almost solely responsible for the observed increase. Lactobacillus OTU4228 constitutes approximately $7 \%$ of the total microbiota in piglets weaned to the basal HC diet (over $85 \%$ of the total Lactobacillus community). This increases to over 21 and $15 \%$ of the total microbiota in piglets weaned to the $\mathrm{HC}+\mathrm{L}$ and $\mathrm{HC}+\mathrm{S}$ diets, respectively (Fig. 1). Although the response of Lactobacillus OTU4228, in terms of increased population abundance, is similar in piglets weaned to diets supplemented with either lactose or saccharin/NHDC, the disparity between caecal lactic acid concentrations suggests that the underlying mechanisms are quite different.

It has been shown that there is a rapid and significant decrease in pig intestinal lactase activity with both age and weaning ${ }^{(43-45)}$, indicating that a substantial amount of ingested lactose may not be digested by the host ${ }^{(46)}$. Lactose is then available as a highly metabolisable substrate readily utilised by bacteria (particularly lactobacilli), initially in the distal regions of the small intestine as well as in the caecum $^{(46)}$. Increases in the population abundance of Lactobacillus have previously been demonstrated in piglets fed diets supplemented with lactose ${ }^{(15,16)}$, primarily due to the metabolism of lactose by lactobacilli.

The highly fermentable nature of lactose is reflected in the large increase in lactic acid concentrations seen here in the caecal contents of piglets weaned to the diet containing lactose (population abundance of lactobacilli increases 2-8-fold; lactic acid increases 10-fold). In contrast, the increase in lactic acid concentrations measured in the caecal contents of piglets weaned to the same diet containing saccharin/ NHDC is in proportion to the increase in the population abundance of Lactobacillus (2.1- and 2.5-fold, respectively). This suggests that, unlike lactose which provides an additional substrate for the growth of lactobacilli and subsequent lactic acid production, this artificial sweetener is not a metabolisable energy source that can be fermented by Lactobacillus populations to produce lactic acid.

The effects of artificial sweeteners on gut microbiota have previously been studied in human subjects. It has been shown that the addition of maltitol, a sugar alcohol, to confectionery significantly enhanced the population abundance of both Bifidobacteria and Lactobacillus ${ }^{(25)}$. However, it is notable that maltitol is a fermentable substrate for these gut microbes $^{(25,47)}$.

In the mammalian intestine, the sweet taste receptor, T1R2-T1R3, expressed in enteroendocrine cells, can detect the presence of sugars and artificial sweeteners. This initiates an intracellular signalling pathway leading to the up-regulation of the intestinal glucose transporter, $\mathrm{Na}^{+} /$glucose co-transporter 1 (SGLT1), and an increased capacity of the gut to absorb glucose $^{(48-50)}$. Likewise, yeasts, such as Saccharomyces cerevisiae, possess mutated glucose transporters ( $\mathrm{Snf} 3$ and Rgt2) that act as transmembrane sweet sensors controlling the expression of hexose transporter proteins in the presence of glucose and other sugars ${ }^{(51,52)}$.

Lactobacilli, and many other enteric bacteria, express multiple sugar transport and metabolic systems that allow them to utilise a variety of carbohydrate substrates and adapt quickly to changes in nutrient availability ${ }^{(53)}$. This versatility is of particular importance in an environment such as the gastrointestinal tract. The predominant sugar transport mechanism in these bacteria is the phosphoenolpyruvate: carbohydrate phosphotransferase system (PTS); with over twenty different PTS systems being identified, each is specific for only one or a few sugars ${ }^{(54)}$. There are also multiple non-PTS sugar transport systems such as non-PTS permeases and $\mathrm{ABC}$ transporters for various poly- and oligosaccharides ${ }^{(54)}$ The vast majority of these systems are regulated in the presence of a specific substrate ${ }^{(53,54)}$.

Extracellular sensing is a key method employed by bacteria in order to respond to changes in their environment such as alterations in $\mathrm{pH}$, chemical composition or nutrient availability. Many of these sensory responses are independent of transport or metabolism, but involve the binding of chemical ligands to membrane-spanning sensory receptors in order to initiate intracellular signalling pathways ${ }^{(55,56)}$. Notably, recent evidence has shown that transcription of genes responsible for utilisation of diverse polysaccharides by enteric Bacteroides species can be directly activated by the recognition of signature oligosaccharide ligands by specific receptors ${ }^{(57,58)}$, demonstrating that these systems play a key role in bacterial ability to sense and utilise polysaccharides in gut ecosystems. In the light of results presented here, we propose that lactobacilli may possess a plasma membraneassociated sweet sensor capable of sensing artificial 
sweeteners and initiating pathways controlling carbohydrate transport and metabolism.

However, the influence of the host on the composition and activity of the gut microbiota is becoming increasingly evident and should not be underestimated. Epithelial factors, such as the secretion of growth-promoting mucosal glycans or toxic inhibitory compounds, have been highly implicated in regulating the composition of the intestinal microbiota ${ }^{(26)}$. Furthermore, differences in microbial community structure between different host species have been proposed to arise from distinct selective pressures imposed from within the gut habitat of the respective host ${ }^{(59)}$. Moreover, host genetic factors have also been shown to contribute in part to gut microbiota composition $^{(27,60)}$

Whatever the underlying mechanism(s), the data presented here show that dietary supplementation with saccharin/NHDC artificial sweetener can alter the gastrointestinal microbiota by positively influencing the population abundance of lactobacilli, commensal bacteria that are able to exert a beneficial effect on gut health, immunity and maturation ${ }^{(39,40)}$.

The identification and characterisation of the underlying mechanism(s) will assist in the design of nutritional strategies aimed at manipulating the pig commensal microbiota, promoting the health of the gut particularly during the critical post-weaning period.

\section{Acknowledgements}

The authors gratefully acknowledge the financial support provided by Pancosma SA.

The authors' contributions are as follows: K. D. and S. P. S.-B. designed the research, analysed the data and wrote the paper; K. D. performed the research; A. D. and N. H. performed the bioinformatic analyses of pyrosequencing data; A. N. and D. B. provided scientific and nutritional advice.

K. D., A. D., N. H. and S. P. S.-B. declare no conflicts of interest. A. N. and D. B. are employees of Pancosma SA.

\section{References}

1. Hooper LV, Wong MH, Thelin A, et al. (2001) Molecular analysis of commensal host-microbial relationships in the intestine. Science 291, 881-884.

2. Hooper LV, Midtvedt T \& Gordon JI (2002) How hostmicrobial interactions shape the nutrient environment of the mammalian intestine. Annu Rev Nutr 22, 283-307.

3. Zoetendal EG, Collier CT, Koike S, et al. (2004) Molecular ecological analysis of the gastrointestinal microbiota: a review. J Nutr 134, 465-472.

4. Bauer E, Williams BA, Smidt $\mathrm{H}$, et al. (2006) Influence of the gastrointestinal microbiota on development of the immune system in young animals. Curr Issues Intest Microbiol 7, $35-51$.

5. Bergman EN (1990) Energy contributions of volatile fatty acids from the gastrointestinal tract in various species. Physiol Rev 70, 567-590.

6. Cummings JH \& Macfarlane GT (1997) Colonic microflora: nutrition and health. Nutrition 13, 476-478.

7. Cuff MA, Dyer J, Jones M, et al. (2005) The human colonic monocarboxylate transporter Isoform 1: its potential importance to colonic tissue homeostasis. Gastroenterology 128, 676-686.

8. Daly K \& Shirazi-Beechey SP (2006) Microarray analysis of butyrate regulated genes in colonic epithelial cells. DNA Cell Biol 25, 49-62.

9. Putaala H, Barrangou R, Leyer GJ, et al. (2010) Analysis of the human intestinal epithelial cell transcriptional response to Lactobacillus acidophilus, Lactobacillus salivarius, Bifidobacterium lactis and Escherichia coli. Benef Microbes 1, 283-295.

10. Lähteinen T, Malinen E, Koort JM, et al. (2010) Probiotic properties of Lactobacillus isolates originating from porcine intestine and feces. Anaerobe 16, 293-300.

11. Lalles J-P, Bosi P, Smidt H, et al. (2007) Nutritional management of gut health in pigs around weaning. Proc Nutr Soc 66, 260-268.

12. Hopwood DE \& Hampson DJ (2003) Interactions between the intestinal microflora, diet and diarrhoea, and their influences on piglet health in the immediate post-weaning period. In Weaning the Pig: Concepts and Consequences, pp. 199-217 [JR Pluske, J Le Dividich and MWA Verstegen, editors]. Wageningen: Wageningen Academic Publishers.

13. Konstantinov SR, Favier CF, Zhu WY, et al. (2004) Microbial diversity studies of the porcine gastrointestinal ecosystem during weaning transition. Anim Res 53, 317-324.

14. Konstantinov SR, Awati AA, Williams BA, et al. (2006) Postnatal development of the porcine microbiota composition and activities. Environ Microbiol 8, 1191-1199.

15. Vente-Spreeuwenberg MAM, Verdonk JMAJ, Bakker GCM, et al. (2004) Effect of dietary protein source on feed intake and small intestinal morphology in newly weaned piglets. Livest Prod Sci 86, 169-177.

16. Pierce KM, Sweeney T, Brophy PO, et al. (2006) The effect of lactose and inulin on intestinal morphology, selected microbial populations and volatile fatty acid concentrations in the gastrointestinal tract of the weaned pig. Anim Sci 82, 311-318.

17. Pierce KM, Callan JJ, McCarthy P, et al. (2007) The interaction between lactose level and crude protein concentration on piglet post-weaning performance, nitrogen metabolism, selected faecal microbial populations and faecal volatile fatty acid concentrations. Anim Feed Sci Technol 132, 267-282.

18. Sterk A, Schlegel P, Mul AJ, et al. (2008) Effects of sweeteners on individual feed intake characteristics and performance in group-housed weanling pigs. J Anim Sci 86, 2990-2997.

19. Konstantinov SR, Awati A, Smidt H, et al. (2004) Specific response of a novel and abundant Lactobacillus amylovoruslike phylotype to dietary prebiotics in the guts of weaning piglets. Appl Environ Microbiol 70, 3821-3830.

20. Loh G, Eberhard M, Brunner RM, et al. (2006) Inulin alters the intestinal microbiota and short-chain fatty acid concentrations in growing pigs regardless of their basal diet. J Nutr 136, 1198-1202.

21. Huang C, Qiao S, Lifa D, et al. (2004) Effects of lactobacilli on the performance, diarrhea incidence, VFA concentration and gastrointestinal microbial flora of weaning pigs. Asian Austral J Anim 17, 401-409.

22. Meyer M, Stenzel U \& Hofreiter M (2008) Parallel tagged sequencing on the 454 platform. Nat Protoc 3, 267-278.

23. Community Register of Feed Additives, pursuant to Regulation (EC) No. 1831/2003, appendices 3 \& 4. http://ec.europa.eu/ food/food/animalnutrition/feedadditives/comm_register_feed_ additives_1831-03.pdf 
24. Beards E, Tuohy K \& Gibson G (2010) A human volunteer study to assess the impact of confectionery sweeteners on the gut microbiota composition. Br J Nutr 104, 701-708.

25. Thabuis C, Herbomez AC, Desailly F, et al. (2012) Prebioticlike effects of SweetPearl ${ }^{\mathrm{TM}}$ maltitol through changes in caecal and fecal parameters. Food Nutr Sci (In the Press).

26. Schluter J \& Foster KR (2012) The evolution of mutualism in gut microbiota via host epithelial selection. PLoS Biol 10, e1001424.

27. Benson AK, Kelly SA, Legge R, et al. (2010) Individuality in gut microbiota composition is a complex polygenic trait shaped by multiple environmental and host genetic factors. Proc Natl Acad Sci U S A 107, 18933-18938.

28. Lin C \& Stahl DA (1995) Taxon-specific probes for the cellulolytic genus Fibrobacter reveal abundant and novel equine-associated populations. Appl Environ Microbiol 61, 1348-1351.

29. Daly K \& Shirazi-Beechey SP (2003) Design and evaluation of group-specific oligonucleotide probes for quantitative analysis of intestinal ecosystems: their application to assessment of equine colonic microflora. FEMS Microbiol Ecol 44, 243-252.

30. Quince C, Lanzén A, Curtis TP, et al. (2009) Accurate determination of microbial diversity from 454 pyrosequencing data. Nat Methods 6, 639-641.

31. Haas BJ, Gevers D, Earl AM, et al. (2011) Chimeric 16S rRNA sequence formation and detection in Sanger and 454pyrosequenced PCR amplicons. Genome Res 21, 494-504.

32. Caporaso JG, Bittinger K, Bushman FD, et al. (2010) PyNAST: a flexible tool for aligning sequences to a template alignment. Bioinformatics 26, 266-267.

33. DeSantis TZ, Hugenholtz P, Larsen N, et al. (2006) Greengenes, a chimera-checked 16S rRNA gene database and workbench compatible with ARB. Appl Environ Microbiol 72, 5069-5072.

34. Price MN, Dehal PS \& Arkin AP (2010) FastTree 2 approximately maximum-likelihood trees for large alignments. PLOS ONE 5, e9490.

35. Edgar RC (2010) Search and clustering orders of magnitude faster than BLAST. Bioinformatics 26, 2460-2461.

36. Wang Q, Garrity GM, Tiedje JM, et al. (2007) Naive Bayesian classifier for rapid assignment of rRNA sequences into the new bacterial taxonomy. Appl Environ Microbiol 73, 5261-5267.

37. Caporaso JG, Kuczynski J, Stombaugh J, et al. (2010) QIIME allows analysis of high-throughput community sequencing data. Nat Methods 7, 335-336.

38. Daly K, Proudman CJ, Duncan SH, et al. (2012) Alterations in microbiota and fermentation products in equine large intestine in response to dietary variation and intestinal disease. Br J Nutr 107, 989-995.

39. Kimura K, McCartney AL, McConnell MA, et al. (1997) Analysis of fecal populations of bifidobacteria and lactobacilli and investigation of the immunological responses of their human hosts to the predominant strains. Appl Environ Microbiol 63, 3394-3398.

40. Blum S \& Schiffrin EJ (2003) Intestinal microflora and homeostasis of the mucosal immune response: implications for probiotic bacteria? Curr Issues Intest Microbiol 4, 53-60.

41. Casla D, Requena T \& Gómez R (1996) Antimicrobial activity of lactic acid bacteria isolated from goat's milk and artisanal cheeses: characteristics of a bacteriocin produced by Lactobacillus curvatus IFPL 105. J Appl Bacteriol 81, $35-41$.
42. Varcoe JJ, Krejcarek G, Busta F, et al. (2003) Prophylactic feeding of Lactobacillus acidophilus NCFM to mice attenuates overt colonic hyperplasia. J Food Prot 66, 457-465.

43. Kelly D, Smyth JA \& McCracken KJ (1991) Digestive development of the early-weaned pig. 1. Effect of continuous nutrient supply on the development of the digestive tract and on changes in digestive enzyme activity during the first week post-weaning. Br J Nutr 65, 169-180.

44. Kelly D, King TP, McFadyen M, et al. (1991) Effect of lactation on the decline of brush border lactase activity in neonatal pigs. Gut 32, 386-392.

45. Marion J, Petersen YM, Romé V, et al. (2005) Early weaning stimulates intestinal brush border enzyme activities in piglets, mainly at the posttranscriptional level. J Pediatr Gastroenterol Nutr 41, 401-410.

46. Kim KI, Benevenga NJ \& Grummer RH (1978) Lactase activity and VFA production in the cecum and colon of pigs fed a corn-soy or $40 \%$ whey diet. J Anim Sci $\mathbf{4 6}$, 1648-1657.

47. Oku T, Akiba M, Lee MH, et al. (1991) Metabolic fate of ingested $\left[{ }^{14} \mathrm{C}\right]-$ maltitol in man. J Nutr Sci Vitaminol 37, 529-544.

48. Dyer J, Daly K, Salmon KS, et al. (2007) Intestinal glucose sensing and regulation of intestinal glucose absorption. Biochem Soc Trans 35, 1191-1194.

49. Margolskee RF, Dyer J, Kokrashvili Z, et al. (2007) T1R3 and gustducin in gut sense sugars to regulate expression of $\mathrm{Na}^{+}$glucose cotransporter 1. Proc Natl Acad Sci US A 104, 15075-15080.

50. Shirazi-Beechey SP, Moran AW, Batchelor DJ, et al. (2011) Glucose sensing and signalling; regulation of intestinal glucose transport. Proc Nutr Soc 70, 185-193.

51. Ozcan S \& Johnston M (1999) Function and regulation of yeast hexose transporters. Microbiol Mol Biol Rev 63, 554-569.

52. Forsberg H \& Ljungdahl PO (2001) Sensors of extracellular nutrients in Saccharomyces cerevisiae. Curr Genet $\mathbf{4 0}$, 91-109.

53. Barrangou R, Azcarate-Peril MA, Duong T, et al. (2006) Global analysis of carbohydrate utilization by Lactobacillus acidophilus using cDNA microarrays. Proc Natl Acad Sci U S A 103, 3816-3821.

54. Kotrba P, Inui M \& Yukawa H (2001) Bacterial phosphotransferase system (PTS) in carbohydrate uptake and control of carbon metabolism. J Biosci Bioeng 92, 502-517

55. Armitage JP \& Sockett RE (1987) Sensory transduction in flagellate bacteria. Ann N Y Acad Sci 510, 9-15.

56. Xu J, Chiang HC, Bjursell MK, et al. (2004) Message from a human gut symbiont: sensitivity is a prerequisite for sharing. Trends Microbiol 12, 21-28.

57. Sonnenburg ED, Sonnenburg JL, Manchester JK, et al. (2006) A hybrid two-component system protein of a prominent human gut symbiont couples glycan sensing in vivo to carbohydrate metabolism. Proc Natl Acad Sci US A 103, 8834-8839.

58. Martens EC, Lowe EC, Chiang H, et al. (2011) Recognition and degradation of plant cell wall polysaccharides by two human gut symbionts. PLoS Biol 9, e1001221.

59. Rawls JF, Mahowald MA, Ley RE, et al. (2006) Reciprocal gut microbiota transplants from zebrafish and mice to germ-free recipients reveal host habitat selection. Cell 127, 423-433.

60. Spor A, Koren O \& Ley R (2011) Unravelling the effects of the environment and host genotype on the gut microbiome. Nat Rev Microbiol 9, 279-290. 